\title{
Effects of a tailored strength training program of the upper limb combined with transcranial direct current stimulation (tDCS) in chronic stroke patients: study protocol for a randomised, double-blind, controlled trial
}

Marie-Hélène Milot $^{1 *} \mathbb{D}$, Stephania Palimeris ${ }^{2,3,4}$, Hélène Corriveau ${ }^{1}$, François Tremblay ${ }^{5}$ and Marie-Hélène Boudrias ${ }^{2,3,4}$

\begin{abstract}
Background: A significant proportion of individuals are left with poor residual functioning of the affected arm after a stroke. This has a great impact on the quality of life and the ability for stroke survivors to live independently. While strengthening exercises have been recommended to improve arm function, their benefits are generally far from optimal due to the lack of appropriate dosing in terms of intensity. One way to address this problem is to develop better tools that could predict an individual's potential for recovery and then adjust the intensity of exercise accordingly. In this study, we aim at determining whether an individualized strengthening program based on the integrity of the corticospinal tract, as reflected in the amplitude of motor evoked potentials (MEPs) elicited by transcranial magnetic stimulation (TMS), in conjunction with transcranial direct current stimulation (tDCS), could lead to more optimal outcomes in terms of arm function in chronic stroke patients.

Methods: This multicentre, double-blinded, randomised controlled trial will aim to recruit 84 chronic stroke patients. Before and after training, participants will undergo a clinical evaluation, assessing motor recovery of the affected arm (Fugl-Meyer Stroke Assessment-FMA) and a TMS evaluation to assess the integrity of the corticospinal tract, as reflected in MEP amplitude. Based on their baseline MEPs amplitude, participants will be stratified into three groups of training intensity levels determined by the one-repetition maximum (1RM); 1) low: 35-50\% 1 RM (MEPs < $50 \mu \mathrm{V}$ ); 2) moderate: 50-65\% 1RM (MEPs 50-120 $\mu \mathrm{V}$ ); and 3) high: 70-80\% 1RM (MEPs > $120 \mu \mathrm{V}$ ). Training will target the affected arm (3 times/week for 4 weeks). In addition, participants will be randomly allocated into two tDCS groups (real vs. sham) and tDCS will be applied in an anodal montage during the exercise.

Discussion: This study will determine whether an individualized strength training intervention in chronic stroke survivors can lead to improved arm function. In addition, we will also determine whether combining anodal tDCS over the lesioned hemisphere with strength training can lead to further improvement in arm function, when compared to sham tDCS.

(Continued on next page)
\end{abstract}

\footnotetext{
* Correspondence: marie-helene.milot@usherbrooke.ca

${ }^{1}$ Faculté de médecine et des sciences de la santé, École de réadaptation, Université de Sherbrooke, 1036 Belvédère sud, Sherbrooke, Québec J1H 4C4, Canada

Full list of author information is available at the end of the article
}

(C) The Author(s). 2019 Open Access This article is distributed under the terms of the Creative Commons Attribution 4.0 International License (http://creativecommons.org/licenses/by/4.0/), which permits unrestricted use, distribution, and reproduction in any medium, provided you give appropriate credit to the original author(s) and the source, provide a link to the Creative Commons license, and indicate if changes were made. The Creative Commons Public Domain Dedication waiver (http://creativecommons.org/publicdomain/zero/1.0/) applies to the data made available in this article, unless otherwise stated. 
(Continued from previous page)

Trial registration: ClinicalTrials.gov Identifier: NCT02915185. Registered September 212016.

Keywords: Stroke, Exercise, Transcranial magnetic stimulation, Randomised controlled trial, Transcranial direct current stimulation, Upper limb

\section{Background}

Stroke is a leading cause of severe long-term disability across the globe $[1,2]$. One of the most common disabling consequences of stroke is residual muscle weakness or paresis of the affected arm $[3,4]$, which has a significant impact on patients' activities of daily life and is a major contributor to reduced quality of life $[5,6]$. Consequently, there has been a move to implement strength training as part of rehabilitation after stroke [7], to increase strength and improve function of the affected arm, even at the chronic stage where gains are still observed [8]. Strength training is commonly used with progressive resistance or repetitive practice and there is evidence demonstrating that it is an effective intervention to improve strength and activity after stroke [7, 9]. Yet, variability is observed between individuals, in which some patients demonstrate significant gains in response to strength training programs while others show either minimal or no benefits $[3,10]$. This brings to question whether the intensity of strength training programs is sufficient to produce a training stimulus and challenge individuals' maximum capacity. Although there is a variety of strengthening protocols prescribed to stroke patients, few studies in the rehabilitation literature assess critical training parameters to address each individual's needs and impairments [11, 12]. A meta-analysis by Coupar et al. [13] found that neurophysiological factors, such as the integrity of the corticospinal tract assessed by non-invasive brain stimulation (NIBS) techniques, were strongly associated with upper limb recovery after a stroke; supporting the use of neurophysiological markers in determining a person's potential for recovery and functional performance. Thus, there is an urgent need to identify valid biomarkers of recovery to design better training interventions for the management of post-stroke disability, notably by adapting programs to meet each individual's capacity in terms of potential for recovery.

Transcranial magnetic stimulation (TMS) is a NIBS technique allowing the identification of biomarkers reflecting the integrity of the corticospinal system after a hemispheric stroke [14]. Stimulation of the motor cortex can elicit motor evoked potentials (MEPs) in contralateral limb muscles, whose presence after a stroke is strongly suggestive of preserved functional projections and potential for recovery in the affected limbs. For instance, Jo et al. [15] reported that the presence of MEPs from both hand motor cortices in the early subacute phase was a good predictor of motor function in patients at 3 months after stroke onset. The combining use of TMS and tractography on 53 patients with intracerebral hemorrhage and severe motor weakness showed that patients in whom MEPs could be elicited in the paretic upper limb, and with a preserved corticospinal tract, had better motor outcomes at 6 months post-stroke [16]. On the other hand, other studies have reported that absence of MEPs in response to high intensity TMS of the ipsilesional motor cortex was associated with poor motor recovery of the upper limb in both the acute and chronic stages post stroke [17-19]. In addition to their ability to predict motor recovery, MEPs can also provide information on an individual response to exercises [20]. Several studies have highlighted the importance of this measure not only to assess corticospinal tract integrity to predict patients' potential for recovery but also to predict their response to exercise [18, 21, 22]. Stinear et al. [18] for example, proposed an algorithm that uses MEPs as a marker for stroke survivors' stratification in terms of exercises prescription to optimise functional recovery. Among the various parameters included in the algorithm, such as DTI and the presence of movements at the thumb and shoulder joints, the absence/presence of MEPs in response to TMS from the ipsilesional motor cortex was considered as a crucial parameter to consider in order to stratify patients based on their recovery potential.

In parallel, recent developments in the management of stroke disability indicate that further gains in function can be obtained when rehabilitation interventions are combined with neurostimulation techniques designed to boost motor excitability and enhance response to exercises [23]. The modulation of cortical excitability by transcranial direct current stimulation (tDCS) has gained particular interest because of its promising effects in neurorehabilitation after stroke [24], which include change in cortical excitability [25-27], enhancement of motor performance and change of movement accuracy and speed $[28,29]$. The technique aims at modulating neuronal excitability using a constant and weak current (1-2 mA) which passes through electrodes placed on the scalp. Depending on the current direction, the stimulation can either enhance (anodal) or depress (cathodal) the excitability of the stimulated cortical area [30]. A number of studies have investigated the impact of anodal tDCS on motor recovery of the affected upper limb of stroke patients [31]. Kim et al. [32] stimulated the ipsilesional cortical region of 10 subacute stroke patients on 
average 12 weeks post infarct and reported significant improvement in motor performance of the hemiparetic hand, outlasting the stimulation session for at least 60 min. Similar results were obtained by Fregni et al. [33] and Hummel et al. [34], where stroke patients were found to have functional improvement in the paretic hand that outlasted the stimulation period with an improvement magnitude of 6.7 and $8.9 \%$, respectively. Furthermore, results from a meta-analysis [35] also support the therapeutic potential of tDCS as an adjuvant treatment strategy to enhance training in stroke patients with upper limb deficits, where tDCS demonstrated a significant impact on rehabilitative training with a moderate effect size of +0.52 $(p<0.001)$ and $+0.69(p<0.001)$ for both immediate and longer-lasting analyses (up to 6 months), respectively.

According to the existing literature, there is a clear need to further validate the use of MEPs as a classification tool and to explore new ways on how to refine the use of this measure, in order to optimize post-stroke training interventions. In addition, since tDCS is still relatively new in the field of stroke rehabilitation, no study to date has tried to determine whether tDCS can enhance the effects of a strength training intervention in stroke patients; a type of intervention commonly used in rehabilitation [3]. Ultimately, there is an urgent need to design better training interventions for the management of post-stroke disability, notably by adapting programs to meet each individual's capacity in terms of potential for recovery. There is also an important need to determine whether non-invasive brain stimulation techniques, such as tDCS, can be used in conjunction with tailored strength training exercises to boost functional recovery after stroke.

The primary goal of this RCT is to determine whether a 4-week tailored strength training program could lead to improved arm function in chronic stroke survivors. The secondary aim is to determine whether combining anodal tDCS of the lesioned hemisphere with strength training could lead to further improvement in arm function when compared to sham tDCS. This study will help determine whether tailored strength training interventions in stroke survivors, based on MEPs amplitude, can lead to greater gains in arm function. In addition, it will also establish whether combining anodal tDCS with tailored strength training can further promote recovery in stroke survivors when compared to sham tDCS.

\section{Methods}

\section{Study design and setting}

This is a multi-centre, randomised controlled trial (RCT) study. Figure 1 illustrates the flow diagram of the study from recruitment and screening for eligible participants to post-training outcome assessments. The RCT takes place in Canada and involves three recruiting sites in two provinces (Québec: Montréal, Sherbrooke; Ontario: Ottawa).
The study is ongoing and is currently during subject recruitment phase (May 2017 till present).

\section{Participants}

Participants will be recruited using several recruitment procedures such as newspaper advertisement, search in medical archives and former patient lists from each site. Potential participants will first be screened over the phone to determine their interest and initial eligibility to participate in the study following a script for early recruitment, after which they will be invited to attend each site, for a screening visit and evaluation. Screening and clinical evaluations will be performed to verify eligibility to participate. At the beginning of the first study visit, written consent will be obtained from each participant and all participants will be informed about the duration and procedure of the study.

\section{Inclusion/exclusion criteria}

Males and females will be eligible to participate in the study if they meet the following criteria: 1 ) are aged 18 years and older; 2) have had solely one stroke; 3 ) be in a chronic stroke phase (>6 months) and 4) have completed their rehabilitation treatment. Participants will be excluded from the study if any of the following criteria are met: 1) a significant spasticity at the affected upper limb (a score $\geq 3$ on the modified Ashworth scale) [36]; 2) a significant pain intensity at the affected upper limb (a score $\geq$ 6/10 on the Visual Analog Pain Scale) [37]; 3) a major sensory deficit (a score $\leq 25 / 34$ on the Nottingham Sensory Assessment) [38]; 3) a presence of hemineglect (> 70\% of unshaded lines on the same side as the motor deficit on the Line Cancellation Test) [39]; 4) an apraxia (a score > 2.5 on the Alexander Test) [40]; 5) any cognitive impairments (a score $\leq 2 / 5$ on the Mini-Cog Test) [41]; 6) the presence of a neurological disorder other than a stroke; 7) concomitant orthopaedic problems at the affected upper limb and 8) any contraindication to TMS and/or tDCS, such as epilepsy, metallic implants, a cardiac pace-maker or pregnancy, for female subjects.

\section{Assessment period}

Prior to and following the intervention period, participants will complete clinical and neurophysiological evaluations. Each evaluation day will last approximately $1.5 \mathrm{~h}$ and both visits will take place in the week before (baseline) and the week after (post-training) the intervention.

\section{Randomisation and blinding}

According to the amplitude of their TMS-induced MEP responses, three levels of stratification will be used, which are adapted from Milot et al. [22]: 1) no detectable MEPs $(<50 \mu \mathrm{V}) ; 2)$ detectable MEPs $(50-120 \mu \mathrm{V})$ and 3) clearly detectable MEPs $(>120 \mu \mathrm{V})$. Within each stratum, participants will be further randomly allocated 


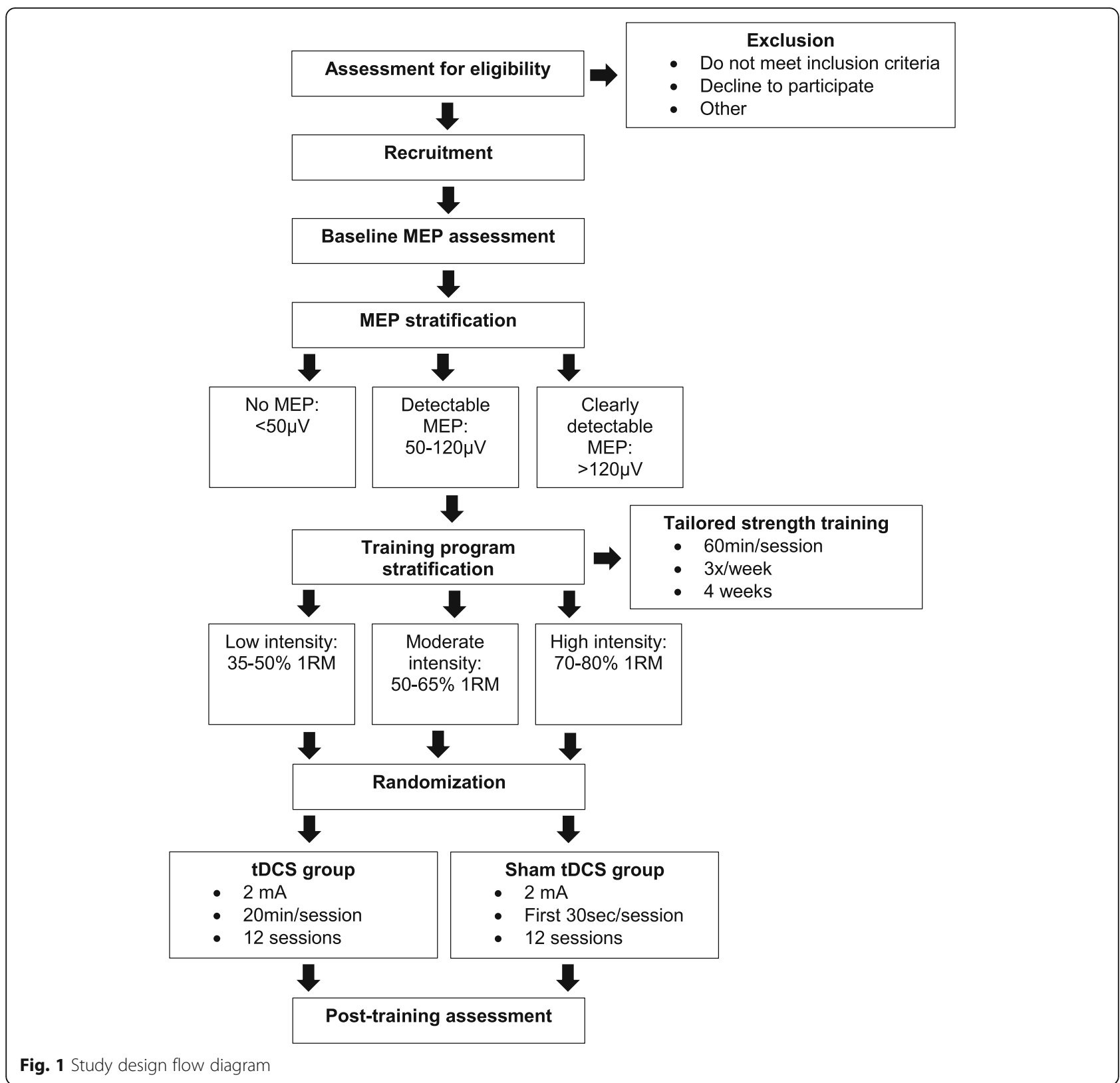

into two tDCS groups: 1) tDCS real group and 2) tDCS sham group. Participants will be randomly assigned to either the tDCS real group or the tDCS sham group with a 1:1 allocation. Randomisation allocation will be generated by a computer using a blocked randomisation with variable block sizes of 2 and 4 . The participants, the evaluators, involved in the clinical and neurophysiological evaluations, the research staff enrolling participants and the data analysts will be blinded to the tDCS group assignment.

\section{Clinical evaluation}

Baseline participant information including age, gender, time since stroke and location of stroke will be collected at the initial visit of each participant by a blinded evaluator to help characterize the sample. The clinical evaluations will consist of measurement of the range of motion of shoulder flexion, elbow flexion and wrist extension of both upper limbs, the Fugl-Meyer Stroke Assessment-FMA (upper limb section; $66=$ normal) [42], the Box and Block Test [43], the Motor Activity Log [44] and measurement of grip strength, measured by the JAMAR ${ }^{\circ}$ hand dynamometer.

\section{Neurophysiological evaluation}

The neurophysiological evaluation will be performed by a trained evaluator blinded to the participants' tDCS group allocation and will consist of assessment of the integrity of the corticospinal tract and cortical reorganization post 
training through the use of motor evoked potentials (MEPs) elicited by TMS. For TMS testing, participants will be seated in an armchair with both hands resting in pronation. The participant's skin will be cleaned with alcohol and surface electromyography (EMG) electrodes will be positioned over the first dorsal interosseous (FDI) of both hands and the extensor carpi radialis (ECR) of the affected arm. Using a TMS system (Magstim 200², Magstim Company, Dyfed, UK) and a 70-mm figure-of-eight coil, we will first determine the hotspot and resting motor threshold (rMT, lowest intensity to evoke reliable MEPs $\geq 50 \mu \mathrm{V}$ ) from the contralesional hemisphere. These coordinates will be used to guide the identification of the optimal location of the ipsilesional primary motor cortex (M1) of the affected hemisphere, when needed. The rMT will then be determined using the Motor Threshold Assessment Tool software (MTAT 2.0; Clinical Researcher, Knoxville, TN, USA). The software allows for fast estimation of motor threshold through the maximum-likelihood strategy based on the PEST (Parameter Estimation by Sequential Testing) algorithm [45]. Next, a series of suprathreshold stimuli $(130 \% \mathrm{rMT}, n=10)$ will be delivered over the hand motor area to elicit MEP in the resting state. We will repeat these procedures for the ipsilesional hemisphere. If no MEPs can be elicited in the affected FDI, the coil will be moved to target the representation of the arm to elicit MEPs in the affected ECR. If peak-to-peak MEP amplitude of the affected ECR does not reach the chosen threshold of $50 \mu \mathrm{V}$, even at the maximum output of the stimulator (100\%), the participant's response will be classified as "MEP absent". After testing in the resting state, corticomotor excitability of the lesioned hemisphere will be tested in the active state. Participants will remain seated in the armchair and will be asked to actively exert a constant force $(20 \%$ of maximal voluntary effort depending on the participant's ability to contract) against a pinch dynamometer on the affected side using a lateral key-pinch with the thumb and index finger. During the contraction (duration $5 \mathrm{~s}$ ), a suprathreshold TMS pulse (130\% of rMT) will be delivered at $3 \mathrm{~s}$ to elicit a facilitated MEP along with a silent period (SP). The SP refers to the interruption of EMG activity in the target muscle arising from spinal and cortical inhibition (GABA $B$ receptors) resulting from the stimulation [46]. Both facilitated MEPs and SP will be measured 5 times with at least $30 \mathrm{~s}$ between trials to allow for recovery.

\section{Intervention period}

All subjects will participate in an outpatient supervised tailored strength training program, which will follow the recommendations of the American Stroke Association (ASA) position on exercise prescription after stroke [47]. Specifically, it will be a 4-week training intervention, performed 3 times per week non-consecutively, with 3 series per exercise of 10 repetitions and a 2-min break in between exercises. The strength training program will be conducted for $1 \mathrm{~h}$ in an outpatient rehabilitation setting and supervised by an experienced trainer who will closely monitor the participants' exercise performance. The training will commence with a 5-min warm-up comprising of active movements of the muscles to be trained. Using free weights, the one-repetition maximum (1RM), which is the maximum amount of weight load an individual can lift for one repetition, will be estimated by the 10RM [48] in order to avoid tendino-muscular injuries and fatigue. The 10RM will be determined for the muscles playing a key role in the functional performance of the upper limb $[49,50]$. These muscles are the wrist extensors and the elbow and shoulder flexors. In addition, the grip muscles of the affected hand will be trained with a JAMAR ${ }^{\circ}$ dynamometer. Participants' maximal grip force will be determined and used to dose the training of the hand muscles and its progression.

As intensity plays a crucial role in response to training, the intensity of the strength training program will be tailored to each participant's potential for recovery based on their baseline MEP amplitudes and gradation of the intensity will follow the ASA recommendation [47] and will be based on the 1RM. This same gradation will be applied for the hand muscles based on the participants' maximal grip force on the JAMAR ${ }^{\circ}$. Thus, for participants in the MEP < $50 \mu \mathrm{V}$ strata (no detectable MEP; low potential for recovery), the training will start at $35 \%$ of the 1RM for each muscle group and will then be increased by $10 \%$ each week to reach $50 \%$ of the $1 \mathrm{RM}$ by week 4 . For participants in the MEP $50-120 \mu \mathrm{V}$ strata (detectable MEP; moderate potential for recovery), the training will start at $50 \%$ of the $1 \mathrm{RM}$ to reach $65 \%$ by week 4 . For the participants in the MEP $\geq 120 \mu \mathrm{V}$ strata (clearly detectable MEP; high potential for recovery), they will train at $70 \%$ of the $1 \mathrm{RM}$ during week 1 and progress to $80 \%$ of the $1 \mathrm{RM}$ at week 4 . In combination to the \% 1RM, the Borg Rating of Perceived Exertion Scale (BRPE), a measure of an individual's self-perceived physical exertion during exercise, will be used to further control the intensity of exercise within and between MEP strata while taking into account the individual's residual ability. The Borg Scale can be accurately rated by chronic stroke survivors and is a valid tool for regulating exercise intensity [51, 52]. Thus, for the first three training weeks, participants in each MEP stratum will train at a perceived intensity between 11 and 13/20 ("fairly light"/"somewhat hard"), corresponding to about $66 \%$ of an individual's maximal effort, and progress toward a perceived intensity between 15 and $17 / 20$ ("hard"/"very hard"), corresponding to about $80 \%$ of an individual's maximal effort, at week 4 .

\section{Transcranial direct current stimulation (tDCS)}

An anodal montage over the ipsilesional hemisphere will be used. The localisation of the lesioned M1 and placement of 
electrodes will follow the protocol from DaSilva et al. [53]. In essence, the vertex will be located by marking the distance halfway between the nasion and inion and the distance between the right/left pre-auricular points. Location of M1, corresponding to C3/C4 on the EEG system, will be estimated by using $20 \%$ of the pre-auricular distance from the vertex. The anode will be placed over the M1 area whereas the cathode will be placed on the contralateral supra-orbital region. For the tDCS group, a direct current will be generated by a tDCS stimulator and gradually increased in a ramp-like fashion over the first $8 \mathrm{~s}$ until a maximum intensity of $2 \mathrm{~mA}$ is achieved. The tDCS will be applied for 20 min during each training session for a total of 12 sessions. The parameters chosen in the proposed project are considered safe for the application of tDCS [30, 31, 54]. For the group receiving sham tDCS, the protocol will be similar to the tDCS real group although the stimulation will be applied for the first $30 \mathrm{~s}$ only; a duration long enough to induce similar perceived sensation as real tDCS, to ensure blindness of the participants to the tDCS type [55]. After each training session, using a home-developed questionnaire, participants will be questioned about the presence of tDCS symptoms, their intensity and relatedness to tDCS.

If any participants develop contra-indications to the training intervention or the tDCS stimulation, they will be discontinued from the intervention but will be included in an intention to treat analysis.

\section{Outcome measures}

\section{Primary outcome measures}

The primary outcome measures will be change in Fugl-Meyer Stroke Assessment Scale-FMA (66 = normal) [42], which will be used to assess changes in the trained arm motor function and change in peak-to-peak MEP amplitude, motor threshold and silent period, elicited by TMS, to assess changes in motor cortex excitability and cortical reorganization.

\section{Secondary outcome measures}

Secondary outcome measures will include:

- change in grip strength, which will be measured by the $\mathrm{JAMAR}^{\circ}$ hand dynamometer (average of 3 trials in $\mathrm{kg}$ );

- change in Box and Block test, which will be used to evaluate manual dexterity by counting the number of blocks that can be moved from one compartment to another in $60 \mathrm{~s} \mathrm{[43];}$

- change in Motor Activity Log, which will assess participants' self-reported level and quality of use of the affected arm in activities of daily living (ADL) [44] and;
- change in active and passive ranges of motion in shoulder flexion, elbow flexion and wrist extension, which will be assessed with a goniometer.

Along with the FMA, these variables were chosen to ensure that the most severely affected participants would be able to perform to some extent the required tasks, knowing that individuals without MEPs often present limited voluntary movement at the affected upper limb.

\section{Sample size and power calculations}

A priori power analysis was performed in G*Power 3.1.9.2, using a two-tailed independent samples t-test having an alpha level of 0.05 , to calculate the sample size required in this study. By stratifying participants based on their MEP amplitude to provide an appropriate dosage of training, we expect all participants within the three MEP strata to benefit from the 4-week tailored strength training program. The sample size was thus calculated based on the expected difference in motor function gains between the tDCS real and the tDCS sham groups. Based on the results of studies having used repetitive application of tDCS in chronic stroke survivors, we expect an 8-point gain in FMA for the tDCS real group [56]. Furthermore, preliminary results based on this study showed a 7-point gain on the FMA scale after 4 weeks of strength training for participants presenting pre-training MEPs. Therefore, we estimate that the tDCS sham group (including participants with and without MEP) will show at least a 6-point gain on the FMA scale, exceeding the 5-point gain minimal detectable change (MDC) of this scale [57]. Thus, with an estimated average difference in FMA gain between both groups of 2 points $(\mathrm{SD}=3)$, with an effect size of 0.66 and a power of $85 \%$, we calculated that a total of 84 participants will be needed to detect differences between groups. However, presuming an attrition rate of $20 \%$, a total of 105 participants will be recruited for the purpose of this study.

\section{Statistical analysis}

Descriptive statistics will be used to characterize the sample. We will verify if, at the beginning, the two tDCS groups will be comparable by using independent $t$-tests or Chi-squared tests, depending on the nature of the variables. To evaluate the impact of tailored exercises based on MEP stratification on changes in FMA and MEP measures, as well as the impact of tDCS on enhancement of training response, a two-way 3X2 ANOVA [MEP strata (three levels) and type of tDCS (2 levels)] will be used. The significance level will be set at 0.05 . If an interaction is noted, paired $t$-tests will be used to locate any significant differences in each stratum with a Bonferroni correction for multiple tests (adjusted $p$-value of 0.02). 


\section{Discussion}

This study will be the first RCT to integrate a commonly used rehabilitation treatment, which is a strength training program of the affected arm, with state-of-the-art brain evaluation protocols and neurostimulation techniques (TMS and tDCS) to allow optimization of the intensity of training based on each stroke survivor's recovery potential. This study will create an initial, yet strong evidence base for MEP stratification as a tool to guide clinicians in providing optimal tailored exercise programs to support recovery post-stroke. More importantly, the marked expected improvement in the affected upper limb's motor function, following the tailored strength training program, will allow stroke survivors to have a more active lifestyle and ultimately optimal quality of life.

\section{Abbreviations}

ADL: Activities of daily living; ASA: American stroke association; BRPE: Borg Rating of Perceived Exertion Scale; ECR: Extensor carpi radialis;

EMG: Electromyography; FDI: First dorsal interosseous; FMA: Fugl-Meyer Stroke Assessment Scale; M1: Primary motor cortex; MDC: Minimal detectable change; MEP: Motor evoked potential; NIBS: Non-invasive brain stimulation; RCT: Randomised controlled trial; RM: Repetition maximum; rMT: Resting motor threshold; SD: Standard deviation; SP: Silent period; tDCS: Transcranial direct current stimulation; TMS: Transcranial magnetic stimulation
\end{abstract}

\section{Acknowledgements}

The authors wish to acknowledge the role of Marie-Claude Girard, Sonia Toy, Dhia Amara and Anthony Ramaud in training the participants.

\section{Funding}

This study has received funding from Brain Canada Foundation, Fonds de recherche du Québec-Santé, Fondation Vitae, Centre de recherche interdisciplinaire en réadaptation du Montréal métropolitain (CRIR) and the Jewish Rehabilitation Hospital. The funding bodies are independent of the design of the study and collection, analysis, and interpretation of data and in writing the manuscript.

\section{Availability of data and materials}

The datasets used and/or analysed during the current study are available from the corresponding author on reasonable request.

\section{Authors' contributions}

MHM conceived the study. MHM, HC, FT and MHB contributed to the design and implementation of the protocol. SP helped with the implementation and drafting of the manuscript. All authors contributed to refinement of the study protocol and approved the final manuscript.

\section{Ethics approval and consent to participate}

This study has been approved by the Research Ethics Committee (REC) of the CIUSSS de I'Estrie-CHUS (MP-22-2016-630) and is responsible for its follow-up. Informed, written consent will be obtained from all participants prior to participation in the study by one of the research staff. Participants will be able to resign at any time without statement of reasons. All data will be anonymously processed.

\section{Consent for publication}

Not applicable.

\section{Competing interests}

The authors declare that they have no competing interests.

\section{Publisher's Note}

Springer Nature remains neutral with regard to jurisdictional claims in published maps and institutional affiliations.

\section{Author details}

'Faculté de médecine et des sciences de la santé, École de réadaptation, Université de Sherbrooke, 1036 Belvédère sud, Sherbrooke, Québec J1H 4C4, Canada. ${ }^{2}$ Faculty of Medicine, School of Physical and Occupational Therapy, McGill University, Montreal, Quebec, Canada. ${ }^{3}$ Feil and Oberfeld Research Centre, BRAIN Lab, Jewish Rehabilitation Hospital, Laval, Quebec, Canada. ${ }^{4}$ Montreal Center for Interdisciplinary Research in Rehabilitation (CRIR), Montreal, Quebec, Canada. ${ }^{5}$ Faculty of Health Sciences, School of Rehabilitation Sciences, University of Ottawa, Ottawa, Ontario, Canada.

Received: 2 May 2019 Accepted: 17 May 2019

Published online: 24 May 2019

\section{References}

1. Feigin VL, Lawes CM, Bennett DA, Barker-Collo SL, Parag V. Worldwide stroke incidence and early case fatality reported in 56 population-based studies: a systematic review. Lancet Neurol. 2009;8(4):355-69.

2. Roger VL, Go AS, Lloyd-Jones DM, Adams RJ, Berry JD, Brown TM, Carnethon MR, Dai S, de Simone G, Ford ES, et al. Heart disease and stroke statistics--2011 update: a report from the American Heart Association. Circulation. 2011;123(4):e18-e209.

3. Patten C, Lexell J, Brown HE. Weakness and strength training in persons with poststroke hemiplegia: rationale, method, and efficacy. J Rehabil Res Dev. 2004:41(3a):293-312.

4. Prabhakaran S, Zarahn E, Riley C, Speizer A, Chong JY, Lazar RM, Marshall RS, Krakauer JW. Inter-individual variability in the capacity for motor recovery after ischemic stroke. Neurorehabil Neural Repair. 2008;22(1):64-71.

5. Fleming MK, Newham DJ, Roberts-Lewis SF, Sorinola IO. Self-perceived utilization of the paretic arm in chronic stroke requires high upper limb functional ability. Arch Phys Med Rehabil. 2014;95(5):918-24.

6. Rondina JM, Park CH, Ward NS. Brain regions important for recovery after severe post-stroke upper limb paresis. J Neurol Neurosurg Psychiatry. 2017; 88(9):737-43.

7. Ada L, Dorsch S, Canning CG. Strengthening interventions increase strength and improve activity after stroke: a systematic review. Aust J Physiother. 2006;52(4):241-8.

8. Allman C, Amadi U, Winkler AM, Wilkins L, Filippini N, Kischka U, Stagg CJ, Johansen-Berg H. Ipsilesional anodal tDCS enhances the functional benefits of rehabilitation in patients after stroke. Sci Transl Med. 2016;8(330): 330 re331.

9. de Sousa DG, Harvey LA, Dorsch S, Glinsky JV. Interventions involving repetitive practice improve strength after stroke: a systematic review. J Physiother. 2018;64(4):210-21.

10. Harris JE, Eng JJ. Strength training improves upper-limb function in individuals with stroke: a meta-analysis. Stroke. 2010;41(1):136-40.

11. Pak S, Patten C. Strengthening to promote functional recovery poststroke: an evidence-based review. Top Stroke Rehabil. 2008;15(3):177-99.

12. Lohse KR, Lang CE, Boyd LA. Is more better? Using metadata to explore doseresponse relationships in stroke rehabilitation. Stroke. 2014;45(7):2053-8.

13. Coupar F, Pollock A, Rowe P, Weir C, Langhorne P. Predictors of upper limb recovery after stroke: a systematic review and meta-analysis. Clin Rehabil. 2012;26(4):291-313.

14. Stinear C. Prediction of recovery of motor function after stroke. Lancet Neurol. 2010;9(12):1228-32

15. Jo JY, Lee A, Kim MS, Park E, Chang WH, Shin Yl, Kim YH. Prediction of motor recovery using quantitative parameters of motor evoked potential in patients with stroke. Ann Rehabil Med. 2016;40(5):806-15.

16. Jang SH, Ahn SH, Sakong J, Byun WM, Choi BY, Chang CH, Bai D, Son SM. Comparison of TMS and DTT for predicting motor outcome in intracerebral hemorrhage. J Neurol Sci. 2010;290(1-2):107-11.

17. Talelli P, Greenwood RJ, Rothwell JC. Arm function after stroke: neurophysiological correlates and recovery mechanisms assessed by transcranial magnetic stimulation. Clin Neurophysiol. 2006;117(8):1641-59.

18. Stinear CM, Barber PA, Smale PR, Coxon JP, Fleming MK, Byblow WD. Functional potential in chronic stroke patients depends on corticospinal tract integrity. Brain. 2007;130(Pt 1:170-80.

19. Bembenek JP, Kurczych K, Karli Nski M, Czlonkowska A. The prognostic value of motor-evoked potentials in motor recovery and functional outcome after stroke - a systematic review of the literature. Funct Neurol. 2012;27(2):79-84.

20. Beaulieu LD, Milot MH. Changes in transcranial magnetic stimulation outcome measures in response to upper-limb physical training in stroke: a 
systematic review of randomized controlled trials. Ann Phys Rehabil Med. 2018;61(4):224-34.

21. Koski L, Mernar TJ, Dobkin BH. Immediate and long-term changes in corticomotor output in response to rehabilitation: correlation with functional improvements in chronic stroke. Neurorehabil Neural Repair. 2004;18(4):230-49.

22. Milot MH, Spencer SJ, Chan V, Allington JP, Klein J, Chou C, PearsonFuhrhop K, Bobrow JE, Reinkensmeyer DJ, Cramer SC. Corticospinal excitability as a predictor of functional gains at the affected upper limb following robotic training in chronic stroke survivors. Neurorehabil Neural Repair. 2014;28(9):819-27.

23. Thibaut A, Chatelle C, Gosseries O, Laureys S, Bruno MA. La stimulation transcrânienne à courant continu : un nouvel outil de neurostimulation. Rev Neurol. 2013;169(2):108-20

24. Webster BR, Celnik PA, Cohen LG. Noninvasive brain stimulation in stroke rehabilitation. NeuroRx. 2006;3(4):474-81.

25. Kubis N. Non-invasive brain stimulation to enhance post-stroke recovery. Front Neural Circuits. 2016;10:56.

26. Tedesco Triccas L, Burridge JH, Hughes AM, Pickering RM, Desikan M, Rothwell JC, Verheyden G. Multiple sessions of transcranial direct current stimulation and upper extremity rehabilitation in stroke: a review and metaanalysis. Clin Neurophysiol. 2016;127(1):946-55.

27. Elsner B, Kwakkel G, Kugler J, Mehrholz J. Transcranial direct current stimulation (tDCS) for improving capacity in activities and arm function after stroke: a network meta-analysis of randomised controlled trials. J Neuroeng Rehabil. 2017;14(1):95.

28. Nitsche MA, Schauenburg A, Lang N, Liebetanz D, Exner C, Paulus W, Tergau F. Facilitation of implicit motor learning by weak transcranial direct current stimulation of the primary motor cortex in the human. J Cog Neurosci. 2003:15(4):619-26.

29. Kobayashi M, Hutchinson S, Theoret H, Schlaug G, Pascual-Leone A. Repetitive TMS of the motor cortex improves ipsilateral sequential simple finger movements. Neurology. 2004;62(1):91-8.

30. Marquez J, van Vliet P, McElduff P, Lagopoulos J, Parsons M. Transcranial direct current stimulation (tDCS): does it have merit in stroke rehabilitation? A systematic review. Int J Stroke. 2015;10(3):306-16.

31. Bastani A, Jaberzadeh S. Does anodal transcranial direct current stimulation enhance excitability of the motor cortex and motor function in healthy individuals and subjects with stroke: a systematic review and meta-analysis. Clin Neurophysiol. 2012;123(4):644-57.

32. Kim DY, Ohn SH, Yang EJ, Park Cl, Jung KJ. Enhancing motor performance by anodal transcranial direct current stimulation in subacute stroke patients. Am J Phys Med Rehabil. 2009:88(10):829-36.

33. Fregni F, Boggio PS, Mansur CG, Wagner T, Ferreira MJ, Lima MC, Rigonatti SP, Marcolin MA, Freedman SD, Nitsche MA, et al. Transcranial direct current stimulation of the unaffected hemisphere in stroke patients. Neuroreport. 2005;16(14):1551-5.

34. Hummel F, Celnik P, Giraux P, Floel A, Wu WH, Gerloff C, Cohen LG. Effects of non-invasive cortical stimulation on skilled motor function in chronic stroke. Brain. 2005;128(Pt 3):490-9.

35. Backhaus WAM, Hummel FC. Transcranial direct current stimulation and its effects on upper extremity neurorehabilitative training in stroke: a metaanalysis. Neurol Dis Stroke Int. 2018;1:1.

36. Katz RT, Rovai GP, Brait C, Rymer WZ. Objective quantification of spastic hypertonia: correlation with clinical findings. Arch Phys Med Rehabil. 1992; 73(4):339-47.

37. Boonstra AM, Schiphorst Preuper HR, Balk GA, Stewart RE. Cut-off points for mild, moderate, and severe pain on the visual analogue scale for pain in patients with chronic musculoskeletal pain. Pain. 2014;155(12):2545-50

38. Lincoln NB, Jackson JM, Adams SA. Reliability and revision of the Nottingham sensory assessment for stroke patients. Physiother. 1998;84(8):358-65.

39. Albert ML. A simple test of visual neglect. Neurology. 1973;23(6):658-64.

40. Alexander MP, Baker E, Naeser MA, Kaplan E, Palumbo C. Neuropsychological and neuroanatomical dimensions of ideomotor apraxia. Brain. 1992;115(Pt 1):87-107.

41. Holsinger T, Plassman BL, Stechuchak KM, Burke JR, Coffman CJ, Williams JW $\mathrm{Jr}$. Screening for cognitive impairment: comparing the performance of four instruments in primary care. J Am Geriatr Soc. 2012;60(6):1027-36.

42. Fugl-Meyer AR, Jaasko L, Leyman I, Olsson S, Steglind S. The post-stroke hemiplegic patient. 1. A method for evaluation of physical performance. Scand J Rehabil Med. 1975;7(1):13-31.
43. Mathiowetz V, Volland G, Kashman N, Weber K. Adult norms for the box and block test of manual dexterity. Am J Occup Ther. 1985;39(6):386-91.

44. Uswatte G, Taub E, Morris D, Light K, Thompson PA. The motor activity Log28: assessing daily use of the hemiparetic arm after stroke. Neurology. 2006; 67(7):1189-94

45. Mishory A, Molnar C, Koola J, Li X, Kozel FA, Myrick H, Stroud Z, Nahas Z, George MS. The maximum-likelihood strategy for determining transcranial magnetic stimulation motor threshold, using parameter estimation by sequential testing is faster than conventional methods with similar precision. J ECT. 2004;20(3):160-5.

46. Rossini PM, Burke D, Chen R, Cohen LG, Daskalakis Z, Di lorio R, Di Lazzaro V, Ferreri F, Fitzgerald PB, George MS, et al. Non-invasive electrical and magnetic stimulation of the brain, spinal cord, roots and peripheral nerves: basic principles and procedures for routine clinical and research application. An updated report from an I.F.C.N. committee. Clin Neurophysiol. 2015: 126(6):1071-107.

47. Billinger SA, Arena R, Bernhardt J, Eng JJ, Franklin BA, Johnson CM, MacKayLyons M, Macko RF, Mead GE, Roth EJ, et al. Physical activity and exercise recommendations for stroke survivors: a statement for healthcare professionals from the American Heart Association/American Stroke Association. Stroke. 2014;45(8):2532-53.

48. Brzycki M. Strength testing - predicting a one-rep max from reps-to-fatigue. J Phys Educ Recreat Dance. 1993;64(1):88-90.

49. Boissy P, Bourbonnais D, Carlotti MM, Gravel D, Arsenault BA. Maximal grip force in chronic stroke subjects and its relationship to global upper extremity function. Clin Rehabil. 1999;13(4):354-62.

50. Mercier C, Bourbonnais D. Relative shoulder flexor and handgrip strength is related to upper limb function after stroke. Clin Rehabil. 2004;18(2):215-21.

51. Wallace AC, Talelli P, Dileone M, Oliver R, Ward N, Cloud G, Greenwood R, Di Lazzaro V, Rothwell JC, Marsden JF. Standardizing the intensity of upper limb treatment in rehabilitation medicine. Clin Rehabil. 2010;24(5):471-8.

52. Milot MH, Leonard G, Corriveau H, Desrosiers J. Using the Borg rating of perceived exertion scale to grade the intensity of a functional training program of the affected upper limb after a stroke: a feasibility study. Clin Interv Aging. 2019;14:9-16.

53. DaSilva AF, Volz MS, Bikson M, Fregni F. Electrode positioning and montage in transcranial direct current stimulation. J Vis Exp. 2011;(51):2744. https:// doi.org/10.3791/2744.

54. Nitsche MA, Liebetanz D, Lang N, Antal A, Tergau F, Paulus W. Safety criteria for transcranial direct current stimulation (tDCS) in humans. Clin Neurophysiol. 2003;114(11):2220-2; author reply 2222-2223.

55. Gandiga PC, Hummel FC, Cohen LG. Transcranial DC stimulation (tDCS): a tool for double-blind sham-controlled clinical studies in brain stimulation. Clin Neurophysiol. 2006;117(4):845-50.

56. Bolognini N, Vallar G, Casati C, Latif LA, El-Nazer R, Williams J, Banco E, Macea DD, Tesio L, Chessa C, et al. Neurophysiological and behavioral effects of tDCS combined with constraint-induced movement therapy in poststroke patients. Neurorehab Neural Repair. 2011;25(9):819-29.

57. Wagner JM, Rhodes JA, Patten C. Reproducibility and minimal detectable change of three-dimensional kinematic analysis of reaching tasks in people with hemiparesis after stroke. Phys Ther. 2008;88(5):652-63.

\section{Ready to submit your research? Choose BMC and benefit from:}

- fast, convenient online submission

- thorough peer review by experienced researchers in your field

- rapid publication on acceptance

- support for research data, including large and complex data types

- gold Open Access which fosters wider collaboration and increased citations

- maximum visibility for your research: over $100 \mathrm{M}$ website views per year

At $\mathrm{BMC}$, research is always in progress.

Learn more biomedcentral.com/submission 